\title{
AUTOMORPHISMS OF FREE METABELIAN GROUPS
}

\author{
BY \\ S. BACHMUTH(1)
}

1. Introduction. Let $F$ denote the free group of rank $n$ and $F^{\prime \prime}$ its second commutator subgroup. Then $\Phi=F / F^{\prime \prime}$ will denote the free metabelian group of rank $n$. In this paper we will be considering those automorphisms of $\Phi$ which leave the elements of $\Phi$ fixed in their commutator class. Instead of constantly repeating this phrase we will call these automorphisms IA-automorphisms. The letters I and A are used to remind the reader that these automorphisms are those which induce the identity automorphism in the abelianized group.

Suppose $a_{1}, a_{2}, \cdots, a_{n}$ is a set of free generators for $\Phi$. Then denoting commutation by square brackets and transformation by exponentiation, a convenient set of generators for $\Phi^{\prime}$, the commutator subgroup of $\Phi$, is given by

$$
\left[a_{i}, a_{j}\right]^{a_{1}^{k_{1}^{1} a_{2}^{k^{2}} \ldots a_{n}^{k^{n}}}}
$$

where $i<j$ and $k_{i}(i=1,2, \cdots, n)$ are arbitrary integers. Because commutators commute in $\Phi^{\prime}$, any element of $\Phi^{\prime}$ may therefore be conveniently written in the form

$$
\left[a_{1}, a_{2}\right]^{P_{1}}\left[a_{1}, a_{3}\right]^{P_{2}} \cdots\left[a_{n-1}, a_{n}\right]^{P_{m}}
$$

where the $P_{j}$ are polynomials in $a_{i}^{ \pm 1}$. Thus any IA-automorphism of $\Phi$ may be described by a mapping of the form

$$
a_{i} \rightarrow a_{i}\left[a_{1}, a_{2}\right]^{P_{1}^{(i)}} \cdots\left[a_{n-1}, a_{n}\right]^{P_{m}^{(i)}} \quad(i=1,2, \cdots, n)
$$

where the $P_{j}^{(i)}$ are polynomials in the (commuting) variables $a_{i}^{ \pm 1}$.

A faithful $2 \times 2$ matrix representation of $\Phi$ given by W. Magnus [5] immediately yields a faithful representation of the group of IA-automorphisms of $\Phi$ by $n \times n$ matrices, the entries of which may be considered as elements in the group ring of a free abelian group of rank $n$. Of course in order that a mapping of the form (1.2) be an automorphism of $\Phi$, there are restrictions on the polynomials $P_{j}^{(i)}$, which in turn yield restrictions on the matrix representing the automorphism. Our first theorem is a necessary and sufficient condition for an $n \times n$ matrix of the

Received by the editors January 7, 1964.

(1) During the research for this paper, the author was supported by NSF grant GP-27. 
type described above to represent an IA-automorphism of $\Phi$. The remainder of the paper is devoted to applications of this representation.

Our first application is to the case where $\Phi$ has rank 2 . In this situation we will show (Theorem 2) that any IA-automorphism of $\Phi$ is an inner automorphism. (The same result for the free group of rank 2 was shown by J. Nielsen [6].) An interesting corollary of this theorem is that any automorphism of $\Phi=F / F^{\prime \prime}$ is induced by an automorphism of the free group $F$. The corresponding question for the case where $\Phi$ has rank greater than 2 is exceedingly difficult to decide. More will be said about this question in a subsequent publication.

The subsequent publication just mentioned will deal in large part with the group of IA-automorphisms of $\Phi$, and because of the above theorem we will limit ourselves to the situation where the rank of $\Phi$ is at least 3 . In this situation (unlike the case where the rank of $\Phi$ is 2) a difficulty will confront us due to the fact that an IA-automorphism of $\Phi$ may have several different representations in the form (1.2). This situation arises because when $\Phi$ has rank greater than 2 , the set (1.1) is not a free set of generators for the commutator subgroup of $\Phi$. For example, the nontrivial relation

$$
J\left(a_{1}, a_{2}, a_{3}\right) \equiv\left[a_{1}, a_{2}\right]^{1-a_{3}}\left[a_{1}, a_{3}\right]^{-1+a_{2}}\left[a_{2}, a_{3}\right]^{1-a_{1}}=1
$$

holds in $\Phi$. The relation (1.3) is known as the Jacobi identity and as is well known, is, in fact, a law in the metabelian variety. We will show (Theorem 3 ) that (1.3) is essentially the only nontrivial relation occurring among the set (1.1). That is, any nontrivial relation for the set (1.1) of generators of the commutator subgroup of $\Phi$ is a product of transforms of the Jacobi products $J\left(a_{i}, a_{j}, a_{k}\right)$.

To describe our final application, let us define the following three automorphisms $k_{i}(i=1,2,3)$ of the free group $F$ with free generators $a_{1}, a_{2}, a_{3}$ :

$$
\begin{array}{rlrl}
k_{i}: a_{j} & \rightarrow a_{i}\left[a_{j}, a_{k}\right], & & i \neq j \neq k \neq i, \\
a_{r} \rightarrow a_{r}, & r \neq i .
\end{array}
$$

Theorem 4 states that the automorphisms $k_{i}(i=1,2,3)$ generate a free group with the $k_{i}$ as free generators. We will not discuss the full significance of this theorem here, but only mention that the $k_{i}$ are a distinguished subset of the only known set of generators of the IA-automorphism group of $F$ (J. Nielsen [7] and W. Magnus [4]). The method of proof-which is to show that the automorphisms of $\Phi$ induced by the $k_{i}$ are free generators of a free group-also shows that the group of IA-automorphisms of the free metabelian group on $n$ generators $(n \geqq 3)$ which are induced by automorphisms of the free group is not a metabelian group (or soluble of any length). This result is in contrast to the case $n=2$, where Theorem 2 above shows that the IA-automorphism group is metabelian.

The contents of this paper as well as the forthcoming sequel have been extracted from my thesis written at New York University, under the guidance of Professor 
W. Magnus. I would like to express my gratitude to Professor Magnus for his generous and critical help and constant guidance at each stage of my work. I would also like to take this opportunity to thank Professor G. Baumslag for his interest in my work and for his many helpful discussions. Finally I would like to thank the referee for his many helpful comments.

2. Notation. Let $G$ be any group. The commutator of any two elements $x, y$ of $G$ will be denoted by square brackets,

$$
[x, y]=x y x^{-1} y^{-1}
$$

and we will write $[x, y, z]$ for $[[x, y], z]$.

We define $x^{y}$ to mean the transform of $x$ by $y$. That is,

$$
x^{y}=y x y^{-1} .
$$

If $A, B$ are subgroups of $G,[A, B]$ is the subgroup generated by all commutators of the form $[a, b]$ where $a \in A, b \in B$. A subgroup $A$ of $G$ is characteristic if $A$ is mapped into itself under all automorphisms of $G$. If $A$ is a characteristic subgroup of $G$, then any automorphism $\alpha$ of $G$ induces an automorphism $\alpha^{\prime}$ of $G / A$. $\alpha^{\prime}(g A)=\alpha(g) A$ for $g \in G$. The following are characteristic subgroups. The commutator subgroup of $G$ is denoted by $G^{\prime}$. It is defined as

$$
G^{\prime}=[G, G] \text {. }
$$

The second commutator subgroup of $G$ is denoted by $G^{\prime \prime}$. It is defined as

$$
G^{\prime \prime}=\left[G^{\prime}, G^{\prime}\right] \text {. }
$$

An IA-automorphism of $G$ is an automorphism of $G$ which induces the identity automorphism in $G / G^{\prime}$. The letters $F$ and $\Phi$ are reserved for the free group and free metabelian group $F / F^{\prime \prime}$, respectively.

3. The representation of the IA-automorphism group of $\Phi$. Let $\Phi=F / F^{\prime \prime}$ be freely generated by $a_{1}, \cdots, a_{n}$. Let $s_{1}, \cdots, s_{n}, t_{1}, \cdots, t_{n}$ be commuting indeterminates. Then $\Phi$ has a faithful representation in $2 \times 2$ matrices given by the correspondence

$$
a_{i} \leftrightarrow\left(\begin{array}{cc}
s_{i} & t_{i} \\
0 & 1
\end{array}\right) \quad(i=1,2, \cdots, n) .
$$

(Magnus [5].) The elements of these matrices lie in the ring obtained from $Z$ by adjoining the commuting indeterminates and their inverses. Now suppose $\alpha$ is an IA-automorphism of $\Phi$; that is, $\alpha$ is described by a mapping of the form (1.2). Then, in terms of the above matrices, one easily checks that $\alpha$ induces the mapping

$$
\left(\begin{array}{cc}
s_{i} & t_{i} \\
0 & 1
\end{array}\right) \rightarrow\left(\begin{array}{cc}
s_{i} & a_{i_{1}} t_{1}+\cdots+a_{i_{n}} t_{n} \\
0 & 1
\end{array}\right) \quad(i=1,2, \cdots, n)
$$

where the $a_{i j}$ are polynomials in $s_{k}^{ \pm 1}(k=1,2, \cdots, n)$ with coefficients in $Z$. 
DEFINITION. To each IA-automorphism $\alpha$ of $\Phi$, we associate the linear mapping $\bar{\alpha}$ defined by

$$
\bar{\alpha}: t_{i} \rightarrow \sum_{j=1}^{n} a_{i j} t_{j} .
$$

That is, we associate with each IA-automorphism of $\Phi$ an automorphism of the free module generated by the $t_{i}$ with the polynomials in the $s_{i}^{ \pm 1}$ as the ring of operators. It is easy to see that this representation is faithful. We next observe that the units of our operator ring are the elements $s_{1}^{j_{1}} s_{2}^{j_{2}} \cdots s_{n}^{j_{n}}$ (and their negatives), where $j_{1}, j_{2}, \cdots, j_{n}$ are arbitrary integers (Crowell and Fox [2], Chapter VIII). Thus for the linear transformation $\bar{\alpha}$ to represent an IA-automorphism of $\Phi$, it is necessary that the determinant of $\bar{\alpha}$ be $\prod_{i=1}^{n} s_{i}^{j_{i}}$. (det $\bar{\alpha}$ cannot be a negative unit since the substitution $s_{i} \rightarrow 1$ for $i=1,2, \cdots, n$ transforms $\bar{\alpha}$ into the identity mapping.) It is easy to see that this condition is not sufficient. The remainder of this section is devoted to finding a necessary and sufficient condition for an $n \times n$ matrix whose entries are polynomials in the $s_{i}^{ \pm 1}$ to represent an IA-automorphism of $\Phi$. This is achieved by means of the following lemma which will be important to us later in another context.

LEMMA 1. Let $S=s_{1}^{j_{1}} s_{2}^{j_{2}} \cdots s_{n}^{j_{n}}$ where $j_{1}, j_{2}, \cdots, j_{n}$ are arbitrary integers. Let $\gamma_{1}, \cdots, \gamma_{n}$ be polynomials in $s_{i}^{ \pm 1}$. Then the matrix

$$
\left[\begin{array}{cc}
S & \sum_{1}^{n} \gamma_{i} t_{i} \\
0 & 1
\end{array}\right]
$$

is a product of matrices

$$
\left(\begin{array}{cc}
s_{i} & t_{i} \\
0 & 1
\end{array}\right)^{ \pm 1}
$$

if and only if the $\gamma_{i}$ satisfy the identity

$$
\sum_{1}^{n} \gamma_{i}\left(1-s_{i}\right)=1-S
$$

Proof $\left({ }^{2}\right)$. (a) The condition is that, under the substitution $t_{i} \rightarrow 1-s_{i}$, the matrix assumes the form

$$
\left(\begin{array}{cc}
S & 1-S \\
0 & 1
\end{array}\right)
$$

Now it is trivial to check that

$$
\left(\begin{array}{cc}
S & 1-S \\
0 & 1
\end{array}\right)\left(\begin{array}{cc}
T & 1-T \\
0 & 1
\end{array}\right)=\left(\begin{array}{cc}
S T & 1-S T \\
0 & 1
\end{array}\right)
$$

(2) This proof is a modification of my original proof suggested by the referee. 
and hence an induction on the length of a product of matrices of the form (3.0) shows that the $\gamma_{j}$ must satisfy condition (3.1).

(b) Assume conversely that the $\gamma_{i}$ of the matrix

$$
\left(\begin{array}{cc}
S & \sum \gamma_{i} t_{i} \\
0 & 1
\end{array}\right)
$$

satisfies condition (3.1). We will show that this matrix is a product of the matrices of the form (3.0). First we notice that it is enough to show it for the case $S=1$, because if $S=\prod_{i=1}^{n} s_{i}^{j_{t}}$, we multiply our matrix by

$$
\prod_{i=1}^{n}\left(\begin{array}{cc}
s_{i} & t_{i} \\
0 & 1
\end{array}\right)^{j_{i}}
$$

and we get a new matrix of the form

$$
\left(\begin{array}{cc}
1 & \sum P_{i} t_{i} \\
0 & 1
\end{array}\right)
$$

But we saw in the proof of part (a) that the $P_{i}$ satisfy condition (3.1) if the $\gamma_{i}$ do. Hence we assume $S=1$ and (3.1) now becomes

$$
\sum_{i=1}^{n} \gamma_{i}\left(1-s_{i}\right)=0
$$

We complete the proof for $n=3$. In this case (3.2) becomes

$$
\gamma_{1}\left(1-s_{1}\right)+\gamma_{2}\left(1-s_{2}\right)+\gamma_{3}\left(1-s_{3}\right)=0 \text {. }
$$

Three solutions of this equation are

$$
\left\{\begin{array} { l } 
{ \gamma _ { 1 } = P ( 1 - s _ { 2 } ) } \\
{ \gamma _ { 2 } = - P ( 1 - s _ { 1 } ) } \\
{ \gamma _ { 3 } = 0 , }
\end{array} \quad \left\{\begin{array} { l } 
{ \gamma _ { 1 } = Q ( 1 - s _ { 3 } ) } \\
{ \gamma _ { 2 } = 0 } \\
{ \gamma _ { 3 } = - Q ( 1 - s _ { 1 } ) , }
\end{array} \quad \left\{\begin{array}{l}
\gamma_{1}=0 \\
\gamma_{2}=R\left(1-s_{3}\right) \\
\gamma_{3}=-R\left(1-s_{2}\right),
\end{array}\right.\right.\right.
$$

where $P, Q, R$ are arbitrary polynomials in $s_{i}^{ \pm 1}(i=1,2,3)$. It is clear that the ideal generated by $1-s_{1}$ and $1-s_{2}$ is a prime ideal and that $1-s_{3}$ is not in this ideal. Hence it follows that $\gamma_{3}$ must be in this ideal. Thus $\gamma_{3}=Q\left(1-s_{1}\right)+R\left(1-s_{2}\right)$ for some polynomials $Q, R$. From this it follows easily that the general solution of the above equation is a sum of the above three solutions. Thus we must show that any matrix product derived from the matrices,

$$
\begin{aligned}
& \left(\begin{array}{cc}
1 & P\left(1-s_{2}\right) t_{1}-P\left(1-s_{1}\right) t_{2} \\
0 & 1
\end{array}\right), \\
& \left(\begin{array}{cc}
1 & Q\left(1-s_{3}\right) t_{1}-Q\left(1-s_{1}\right) t_{3} \\
0 & 1
\end{array}\right), \\
& \left(\begin{array}{cc}
1 & R\left(1-s_{3}\right) t_{2}-R\left(1-s_{2}\right) t_{3} \\
0 & 1
\end{array}\right),
\end{aligned}
$$


is a product of matrices of the form (3.0). Consider the mapping $\tau$ from $\Phi^{\prime}$ into the free $Z\left[s_{1}^{ \pm 1}, \cdots, s_{n}^{ \pm 1}\right]$-module $M$ spanned by $t_{1}, t_{2}, \cdots, t_{n}$ defined as follows:

$$
\tau\left(\left[a_{i}, a_{j}\right]^{f\left(a_{1}, \ldots, a_{n}\right)}\right)=\left(\left(1-s_{j}\right) t_{i}-\left(1-s_{i}\right) t_{j}\right) f\left(s_{1}, \cdots, s_{n}\right) .
$$

Since the Magnus matrix representation of $\Phi$ gives

$$
\left[a_{i}, a_{j}\right]^{a_{1}^{1_{1}^{1}} a_{2}^{l_{2}^{2}} \cdots a_{n}^{l^{n}}} \leftrightarrow\left(\begin{array}{cc}
1 & s_{1}^{l_{1}} s_{2}^{l_{2}} \cdots s_{n}^{l_{n}}\left(1-s_{j}\right) t_{i}-s_{1}^{l_{1}} \cdots s_{n}^{l_{n}}\left(1-s_{i}\right) t_{j} \\
0 & 1
\end{array}\right),
$$

the proof of Lemma 1 amounts to showing that the following sequence is exact:

$$
0 \rightarrow \Phi^{\prime} \stackrel{\tau}{\rightarrow} M \stackrel{\lambda}{\rightarrow} Z\left[s_{1}^{ \pm 1}, \cdots, s_{n}^{ \pm 1}\right],
$$

where $\lambda$ is defined by the substitution $t_{i} \rightarrow 1-s_{i}$. From (3.3) it is immediate that $\lambda \tau=0$. On the other hand suppose that $f=\sum c_{i}\left(s_{1}, \cdots, s_{n}\right) t_{i}$ is in the kernel of $\lambda$. If we denote $1-s_{i}$ by $\sigma_{i}$, this means that $\sum c_{i}\left(\sigma_{i}, \cdots, \sigma_{n}\right) \sigma_{i}=0$. Think of the $c_{i}$ written out as a sum of monomials $a_{i}$ with coefficients \pm 1 . The proof now proceeds by induction on the total number of monomials in the $c_{i}$. If $f \neq 0$, the hypothesis implies that, for some $i \neq j, c_{i}$ has a term $a_{i}$ and $c_{j}$ a term $a_{j}$ such that $a_{i} \sigma_{i}+a_{j} \sigma_{j}=0$. But then, for some $b, a_{i}=b \sigma_{j}$ and $a_{j}=-b \sigma_{i}$. Now if the total number of the unit monomials is 2 , and thus consists only of $a_{i}$ and $a_{j}$, then

$$
\tau\left(\left[a_{i}, a_{j}\right]^{b}\right)=b \sigma_{j} t_{i}-b \sigma_{i} t_{j},
$$

which shows that $f$ is in the image of $\tau$. Hence we have a basis for induction. If now the $c_{i}$ contain more than 2 monomials, then subtracting $b \sigma_{j} t_{i}-b \sigma_{i} t_{j}$ from $f$ gives a new $f^{\prime}$ in which the total number of unit monomials in the $c_{i}$ is smaller and hence the induction hypothesis can be applied. This completes the proof of Lemma 1.

As a corollary to Lemma 1, we have

THEOREM 1. A necessary and sufficient condition for an $n \times n$ matrix $\alpha=\left(a_{i j}\right)$ (where the $a_{i j}$ are polynomials in the $s_{i}^{ \pm 1}(i=1, \cdots, n)$ ) to represent an $I A$ automorphism of the free metabelian group of rank $n$ is that

(1) $\operatorname{det} \alpha=\prod_{i=1}^{n} s_{i}^{j_{i}}$, where $j_{1}, \cdots, j_{n}$ are integers, and

(2) $\sum_{j=1}^{n} a_{i j}\left(1-s_{j}\right)=1-s_{i}$.

Proof. (1) The transformation must be invertible and (2) it must map the generators of $\Phi$ into matrices representing elements of $\Phi$.

4. The 2-generator group-Nielsen's theorem. In this section $\Phi$ is the free metabelian group on two free generators $a_{1}, a_{2}$. We will prove that the group of IA-automorphisms of $\Phi$ is the group of inner automorphisms of $\Phi$. As previously noted, J. Nielsen proved the same theorem for the free group of rank 2 .

For the 2-generator group we have a considerable simplification of our automorphism and corresponding determinant. Any IA-automorphism $\alpha$ can now be described by the mapping 


$$
\alpha: \begin{aligned}
& a_{1} \rightarrow a_{1}\left[a_{1}, a_{2}\right]^{A} \\
& a_{2} \rightarrow a_{2}\left[a_{1}, a_{2}\right]^{B}
\end{aligned}
$$

where $A$ and $B$ are now polynomials in $a_{1}^{ \pm 1}$ and $a_{2}^{ \pm 1}$. In matrix form $\alpha$ becomes

$$
\alpha=\left(\begin{array}{ll}
P & R \\
Q & S
\end{array}\right)=\left(\begin{array}{cc}
1+s_{1}\left(1-s_{2}\right) A & s_{2}\left(1-s_{2}\right) B \\
-s_{1}\left(1-s_{1}\right) A & 1-s_{2}\left(1-s_{1}\right) B
\end{array}\right)
$$

and

$$
\operatorname{det} \alpha=1+s_{1}\left(1-s_{2}\right) A-s_{2}\left(1-s_{1}\right) B=s_{1}^{j_{1}} s_{2}^{j_{2}} .
$$

Using these preliminary remarks, we now prove the theorem.

THEOREM 2. Any IA-automorphism of $\Phi$ is an inner automorphism.

Proof. One readily verifies that the inner automorphisms which are transformations by $a_{1}$ (or $a_{2}$ ) have determinant equal to $s_{1}$ (or $s_{2}$ ). Thus, we see that there exists an inner automorphism with arbitrary determinant $\left(s_{1}^{j_{1}} s_{2}^{j_{2}}\right)$, whence it suffices to consider only an IA-automorphism $\alpha$ with $\operatorname{det} \alpha=1$. The equation (4.3) for $\operatorname{det} \alpha$ now gives $s_{1}\left(1-s_{1}\right) A=s_{2}\left(1-s_{2}\right) B$, whence, for some $C, A=s_{2}\left(1-s_{1}\right) C$, $B=s_{1}\left(1-s_{2}\right) C$. Consider now the following matrix:

$$
X=\left(\begin{array}{cc}
1 & -s_{1} s_{2}\left(1-s_{2}\right) C t_{1}+s_{1} s_{2}\left(1-s_{1}\right) C t_{2} \\
0 & 1
\end{array}\right)
$$

By Lemma 1, $X$ represents an element of $\Phi$. Let us denote by $\beta$ the inner automorphism which is transformation by $X$. We will complete the proof of Theorem 2 by showing that $\beta \alpha=1$. $\beta \alpha$ is the mapping

$\beta \alpha:$

$$
\begin{aligned}
\left(\begin{array}{cc}
s_{1} & t_{1} \\
0 & 1
\end{array}\right) & \rightarrow X\left(\begin{array}{cc}
s_{1} & P t_{1}+Q t_{2} \\
0 & 1
\end{array}\right) X^{-1} \\
& =\left(\begin{array}{cc}
s_{1} & {\left[P-s_{1} s_{2}\left(1-s_{1}\right)\left(1-s_{2}\right) C\right] t_{1}+\left[Q+s_{1} s_{2}\left(1-s_{1}\right)^{2} C\right] t_{2}} \\
0 & 1
\end{array}\right),
\end{aligned}
$$

$$
\begin{aligned}
\left(\begin{array}{rr}
s_{2} & t_{2} \\
0 & 1
\end{array}\right) & \rightarrow X\left(\begin{array}{cc}
s_{2} & R t_{1}+S t_{2} \\
0 & 1
\end{array}\right) X^{-1} \\
& =\left(\begin{array}{cc}
s_{2} & {\left[R-s_{1} s_{2}\left(1-s_{2}\right)^{2} C\right] t_{1}+\left[S+s_{1} s_{2}\left(1-s_{1}\right)\left(1-s_{2}\right) C\right] t_{2}} \\
0 & 1
\end{array}\right),
\end{aligned}
$$

where $P, Q, R, S$ are defined by (4.2). But since

$$
\begin{aligned}
& P=1+s_{1}\left(1-s_{2}\right) A=1+s_{1} s_{2}\left(1-s_{1}\right)\left(1-s_{2}\right) C, \\
& Q=-s_{1}\left(1-s_{1}\right) A=-s_{1} s_{2}\left(1-s_{1}\right)^{2} C
\end{aligned}
$$

we have 


$$
P-s_{1} s_{2}\left(1-s_{1}\right)\left(1-s_{2}\right) C=1, \quad Q+s_{1} s_{2}\left(1-s_{1}\right)^{2} C=0 .
$$

Similarly,

$$
R-s_{1} s_{2}\left(1-s_{2}\right)^{2} C=0, \quad S+s_{1} s_{2}\left(1-s_{1}\right)\left(1-s_{2}\right) C=1 .
$$

Thus $\beta \alpha$ is the identity mapping and this completes the proof of Theorem 2 .

As a corollary we have

Corollary. Any automorphism of the free metablelian group of rank 2 is induced by an automorphism of the free group of rank 2 .

To the best of my knowledge, this corollary is still an open question for the $n$-generator group $(n \geqq 3)$.

5. A free basis for the commutator subgroup of the free metabelian group. In this section we will show that the only nontrivial relations occurring among the set of commutators (1.1) are products of transforms of Jacobi products. A special case of this theorem is that the set (1.1) is a free set when the rank of $\Phi$ is 2 .

THEOREM 3. In the free metabelian group with free generators $a_{1}, \cdots, a_{n}$, the only nontrivial relations involving commutators of the form

$$
\left[a_{i}, a_{j}\right]^{P},
$$

where $P$ is a polynomial in the commuting variables $a_{k}^{ \pm 1}(k=1,2, \cdots, n)$, are products of transforms of Jacobi products (1.3).

Proof $\left({ }^{3}\right)$. That such products are equal to the identity is well known and is a straightforward check. We will show that the converse is true. Suppose, if possible, that a nontrivial "formal product"

$$
P=\prod_{i<j}\left[a_{i}, a_{j}\right]^{f_{i j}\left(a_{1}, \ldots, a_{n}\right)}
$$

represents the trivial element of $\Phi$, but the total number $t$ of unit monomials in the $f_{i j}$ cannot be shortened (by cancellation or) by use of the Jacobi identity. We may suppose some $f_{l j} \neq 0$. Now let $\tau$ denote the mapping of $\Phi^{\prime}$ into $M$ defined by (3.3) of $\S$. Then $\tau(P)=\Sigma C_{l} t_{i}$, where, in particular,

$$
C_{l}=\sum_{j>l} f_{l j}\left(s_{1}, \cdots, s_{n}\right)\left(1-s_{j}\right)-\sum_{i<l} f_{i l}\left(s_{1}, \cdots, s_{n}\right)\left(1-s_{i}\right) .
$$

Let us change variables by setting $\sigma_{\mu}=1-s_{\mu}$ (and suppose that $t$ was calculated with respect to the corresponding $\left.\chi_{\mu}=1-a_{\mu}\right)$. Then

$$
C_{l}=\sum_{j>l} f_{l j}^{\prime}\left(\sigma_{1}, \cdots, \sigma_{n}\right) \sigma_{j}-\sum_{i<l} f_{i l}^{\prime}\left(\sigma_{1}, \cdots, \sigma_{n}\right) \sigma_{i} .
$$

But $P$ trivial implies that $C_{l}=0$. Thus one of two possibilities must occur:

(3) The proof given is not my original proof, but one communicated to me by the referee. 
(a) For some $j$ and $k, l<j<k, f_{l j}^{\prime}$ contains a monomial $b \sigma_{k}$ and $f_{l k}^{\prime}$ contains $-b \sigma_{j}$, or

(b) For some $j$ and $i, i<1<j, f_{l j}^{\prime}$ contains a monomial $b \sigma_{i}$ and $f_{i l}^{\prime}$ contains $b \sigma_{j}$. In the first case, subtracting

$$
J\left(a_{l}, a_{j}, a_{k}\right)^{b}=\left[a_{l}, a_{j}\right]^{b \chi_{k}}\left[a_{l}, a_{k}\right]^{-b \chi_{j}}\left[a_{j}, a_{k}\right]^{b \chi_{l}}
$$

from $P$ gives a new product $p^{\prime}$ with $t^{\prime}<t$, thus contradicting the assumption that $t$ cannot be shortened by use of the Jacobi identity. Similarly, in the second case subtract

$$
J\left(a_{i}, a_{l}, a_{j}\right)^{b}=\left[a_{i}, a_{l}\right]^{b \chi_{j}}\left[a_{i}, a_{j}\right]^{-b \times l}\left[a_{l}, a_{j}\right]^{b \times i}
$$

from $P$. This completes the proof of the theorem.

My original proof was based upon the observation that any mapping of the form

$$
\begin{array}{lll}
a_{i} \rightarrow a_{i}\left[a_{j}, a_{k}\right]^{f_{j k}} & , & i \neq j \neq k \neq i, \\
a_{r} \rightarrow a_{r}, & r \neq i,
\end{array}
$$

is always an automorphism with determinant equal to 1 regardless what the polynomial $f_{j k}$ is. Hence this immediately leads to conditions on the remaining exponents $f_{\mu v}(\mu \neq j, v \neq k)$ which easily lead to the conclusion that $P$ consists of transforms of Jacobi products. (In the case of more than three generators, repeated use of the above observation must be invoked.) This observation about the mapping (5.1) is very fruitful in another context and will be important in a later work.

It has already been mentioned that Theorem 3 is important in the further study of automorphism groups of free metabelian groups. However, this theorem is one concerning the free metabelian group itself and it is therefore hoped will be an aid in a further study of these important groups. Of course other sets of generators for the commutator subgroup are known which are actually free sets, but these sets are not as convenient to work with as the set (1.1). (All free sets known to me either contain commutators of arbitrary length, or else contain elements inside the brackets which must be raised to arbitrarily high powers.) One might also mention that Theorem 3 can be used to give another solution to the word problem for free metabelian groups.

A converse question to Theorem 3 has been investigated in [8].

6. A free subgroup of the IA-automorphism group of a free group of rank $\geqq 3$. Let $F$ denote a free group. We will first limit ourselves to the case where $F$ has three free generators $a_{1}, a_{2}, a_{3}$ and prove our theorem for this special case. The proof will be more transparent in this situation and afterwards we will state what the generalization is for the case where $F$ has rank $n$.

We will have need for the following criterion due to Malcev. (See Kurosh $\left[3\right.$, p. 38] where it is shown that the $e_{\alpha}$ under "adjoint" multiplication are free 
generators of a free group.) Another proof of the following criterion can be found in Chandler [1] where use is made of the fact that the Malcev ring is a homomorphic image of the Magnus ring.

Criterion. Let $1, \chi_{1}, \chi_{2}, \cdots, \chi_{k}$ be elements in a ring with the relations $\chi_{i}^{2}=0$ for $i=1,2, \cdots, k$. Let $W$ be any reduced word of length $n$ in the $\chi_{i}$ for which no adjacent factors are equal; i.e., there is no factor of the form $\chi_{i}^{l}(l \geqq 2)$ in $W$. Then the elements $z_{i}=1+\chi_{i}(i=1,2, \cdots, k)$ are free generators of a free group under ring multiplication if for all $W$ the following holds: $W$ is not a linear combination of words of length smaller than $n$.

We are going to apply this criterion to the automorphisms $k_{i}(i=1,2,3)$ of $F$ (as defined in (1.4)) when considered as automorphisms of $\Phi$. That is, we are going to show that the automorphisms $k_{i}^{*}$ of $\Phi$ which are induced by the automorphisms $k_{i}$ of $F$ generate a free group with the $k_{i}^{*}$ as free generators. This of course implies that the $k_{i}$ generate a free group with the $k_{i}$ as free generators. For definiteness, we will assume that $j<k$ in the definition of the $k_{i}$. Then one may easily verify that $k_{1}^{*}=I+X, k_{2}^{*}=I+Y, k_{3}^{*}=I+Z$, where $I$ is the identity matrix and

$$
\begin{aligned}
& X=\left[\begin{array}{ccc}
0 & 0 & 0 \\
s_{1}\left(1-s_{3}\right) & 0 & 0 \\
-s_{1}\left(1-s_{2}\right) & 0 & 0
\end{array}\right], \quad Y=\left[\begin{array}{ccc}
0 & s_{2}\left(1-s_{3}\right) & 0 \\
0 & 0 & 0 \\
0 & -s_{2}\left(1-s_{1}\right) & 0
\end{array}\right], \\
& Z=\left[\begin{array}{ccc}
0 & 0 & s_{3}\left(1-s_{2}\right) \\
0 & 0 & -s_{3}\left(1-s_{1}\right) \\
0 & 0 & 0
\end{array}\right] .
\end{aligned}
$$

THEOREM 4. $\left\{k_{1}, k_{2}, k_{3}\right\}$ is a free subgroup (with the $k_{i}$ as free generators) of the IA-automorphism group of $F$.

Proof. We will apply the above criterion to conclude that the $k_{i}^{*}$ are free generators of a free group. Clearly $X^{2}=Y^{2}=Z^{2}=0$. Next, consider a reduced word $W$ in $X, Y, Z$ of length $n$ for which a factor $X^{l}, Y^{l}$, or $Z^{l}(l \geqq 2)$ does not occur. The matrices $X, Y, Z$ have the property that, if $W$ has length $n$, all the nonzero entries in $W$ have degree at least $n$ in the $s_{i}$. Thus the only possibility for $W$ to be a linear combination of words of smaller length is that $W=0$. We claim that $W$ cannot be zero. The proof is by induction on the length of a word. For words of length 2 , we see that $W \neq 0$, since $X Y, Y X, X Z, Z X, Y Z$, and $Z Y$ are all different from zero. Hence we have a basis for induction. Assume $W$ of length $n$ is not zero. We show that increasing the length of $W$ by one results in a new word not zero. There are three cases to consider.

Case 1. $W$ ends in $X$. 


$$
W=\hat{W} X=\left(\hat{w}_{i j}\right)\left[\begin{array}{ccc}
0 & 0 & 0 \\
s_{1}\left(1-s_{3}\right) & 0 & 0 \\
-s_{1}\left(1-s_{2}\right) & 0 & 0
\end{array}\right]=\left[\begin{array}{lll}
a & 0 & 0 \\
b & 0 & 0 \\
c & 0 & 0
\end{array}\right] \text {. }
$$

Our induction hypothesis is that at least one of the entries $a, b, c$ is different from zero. There are only two possibilities of increasing the length of $W$ by one-namely, multiplication by $Y$ or multiplication by $Z$. In these cases we have

or

$$
\left[\begin{array}{lll}
a & 0 & 0 \\
b & 0 & 0 \\
c & 0 & 0
\end{array}\right]\left[\begin{array}{ccc}
0 & s_{2}\left(1-s_{3}\right) & 0 \\
0 & 0 & 0 \\
0 & -s_{2}\left(1-s_{1}\right) & 0
\end{array}\right]
$$

$$
\left[\begin{array}{lll}
a & 0 & 0 \\
b & 0 & 0 \\
c & 0 & 0
\end{array}\right]\left[\begin{array}{ccc}
0 & 0 & s_{3}\left(1-s_{2}\right) \\
0 & 0 & -s_{3}\left(1-s_{1}\right) \\
0 & 0 & 0
\end{array}\right]
$$

In either case, the resulting matrix is different from zero no matter which of $a, b$ or $c$ is different from zero.

Cases 2 and 3. $W$ ends in $Y$ or $W$ ends in $Z$.

In both cases the analysis is similar to that for Case 1 . This completes the proof of Theorem 4.

One obvious extension of this theorem to the case of the free group of rank $n$ with free generators $a_{1}, a_{2}, \cdots, a_{n}$ is to consider $n$ automorphisms $k_{1}, \cdots, k_{n}$ such that the induced automorphisms $k_{i}^{*}$ are of the form $I+X_{i}$, where the matrices $X$ are zero everywhere except in the nondiagonal entries of the $i$ th column. But each nondiagonal entry in this column must be nonzero. Then the above proof is valid and shows that the $k_{1}, \cdots, k_{n}$ are free generators of a free group. It is a simple matter to find $n$ IA-automorphisms satisfying these conditions. One need only multiply automorphisms of the form (1.4) to produce automorphisms which leave all but the $i$ th generator unchanged while the $i$ th generator is mapped into itself times a product of commutators which contain all the remaining generators. For example, if $n=4$, one may take $k_{i}$ as the automorphism which leaves $a_{j}, a_{k}, a_{l}$ unchanged and $a_{i} \rightarrow a_{i}\left[a_{j}, a_{k}\right]\left[a_{j}, a_{l}\right](i \neq j \neq k \neq l \neq i)$. Thus for the free group of rank $n$, the statement of Theorem 4 becomes

THEOREM 4.' For the free group of rank $n$, the subgroup generated by the automorphisms of the form (1.4) contains a free subgroup of rank $n$.

COROLlARY. The IA-automorphism group of the free metabelian group of rank $n(n \geqq 3)$ contains a free subgroup of rank $n$. 


\section{REFERENCES}

1. B. Chandler, The representation of a generalized free product in sin associative ring, Thesis, New York University, New York, 1961.

2. R. Crowell and R. H. Fcx, Introduction to knot theory, Ginn, Boston, 1963.

3. A. G. Kurosh, The theory of groups, Vol. 2, Chelsea, New York, 1956.

4. W. Magnus, Uber n-dimensionale Gittertransformationen, Acta Math. 64(1935), 353-367.

5. - On a theorem of Marshall Hall, Ann. of Math. (2) 40 (1939), 764-768.

6. J. Nielsen, Die Isomorphismen der allgemeinen, unendlicher Gruppe mit zwei Erzeugenden, Ma th. Ann. 78 (1917), 385-397.

7. —_, Die Isomorphismengruppe der freien Gruppen, Math. Ann. 91 (1924), 169-209.

8. S. Bachmuth and J. Lewin, The Jacobi identity in groups, Math. Z. 83 (1964), 170-176.

FAIRLEIGH Dickinson University,

MADISON, NeW JERSEY 